\section{Funding}

G.T. is supported in relation to a National Institute for Health Research (NIHR) Applied Programme grant awarded to the South London and Maudsley NHS Foundation Trust, and in relation to the NIHR Specialist Mental Health Biomedical Research Centre at the Institute of Psychiatry, King's College London and the South London and Maudsley NHS Foundation Trust.

\section{References}

1 Crowley Jr WF, Sherwood L, Salber P, Scheinberg D, Slavkin H, Tilson H, et al. Clinical research in the United States at a crossroads: proposal for a novel public-private partnership to establish a national clinical research enterprise. JAMA 2004; 291: 1120-6.

2 Proctor EK, Landsverk J, Aarons G, Chambers D, Glisson C, Mittman B. Implementation research in mental health services: an emerging science with conceptual, methodological, and training challenges. Adm Policy Ment Health 2009; 36: 24-34.

3 Roen $\mathrm{K}$, Arai L, Roberts $\mathrm{H}$, Popay J. Extending systematic reviews to include evidence on implementation: methodological work on a review of community-based initiatives to prevent injuries. Soc Sci Med 2006; 63: 1060-71.
4 Zazzali JL, Sherbourne C, Hoagwood KE, Greene D, Bigley MF, Sexton TL. The adoption and implementation of an evidence based practice in child and family mental health services organizations: a pilot study of functional family therapy in New York State. Adm Policy Ment Health 2008; 35: 38-49.

5 Grimshaw JM, Eccles MP, Greener J, MacLennan G, Ibbotson T, Kahan JP, et al. Is the involvement of opinion leaders in the implementation of research findings a feasible strategy? Implement Sci 2006; 1: 3.

6 Solberg LI. Recruiting medical groups for research: relationships, reputation, requirements, rewards, reciprocity, resolution, and respect. Implement Sci 2006; 1: 25.

7 Perkins MB, Jensen PS, Jaccard J, Gollwitzer P, Oettingen G, Pappadopulos $E_{\text {‘ }}$ et al. Applying theory-driven approaches to understanding and modifying clinicians' behavior: what do we know? Psychiatr Serv 2007; 58: 342-8.

8 Bowman CC, Sobo EJ, Asch SM, Gifford AL. Measuring persistence of implementation: QUERI Series. Implement Sci 2008; 3: 21.

9 Glisson C, Schoenwald SK, Kelleher K, Landsverk J, Hoagwood KE, Mayberg S, et al. Therapist turnover and new program sustainability in mental health clinics as a function of organizational culture, climate, and service structure. Adm Policy Ment Health 2008; 35: 124-33.

10 Mendel $P$, Meredith LS, Schoenbaum M, Sherbourne CD, Wells KB. Interventions in organizational and community context: a framework for building evidence on dissemination and implementation in health services research. Adm Policy Ment Health 2008; 35: 21-37.

\title{
Professor Freud and hysteria
}

Brener and Freud fourteen years ago, endeavoured to show that in every case of hysteria the illness was traceable to an actual but forgotten occurrence, in most cases to a painful emotional shock or mental trauma, the circumstances of the forgotten occurrence being in most cases discoverable in hypnosis. That is, as Freud said, "the hysterical suffer from reminiscences," or in Boris Sidia's words, from "resurrected moments." They further stated that when this buried reminiscence was translated into the awakened consciousness of the subject, and the subject had given to this recollection its adequate (motor) expression, the hysterical symptoms disappeared. This statement contains an implication which has since undergone considerable development at Freud's hands and forms one of the main props of his argument; he maintains that at the time of the original psychic trauma an adequate and fitting individual reaction was denied the subject, either through force of circumstances or by its voluntary suppression on the subject's part, and that as the consequence of this denied relief, and the voluntary suppression or displacement (Verdrängung) from the field of conscious mentality of the painful impression with its persistent affective colouring, these latter take on an independent, "split-off," subconscious existence, making their own hidden associations and resulting in a more or less fixed psychic dissociation. According to Freud and others, this split-off complex is inaccessible to the waking consciousness, though it may be revived in hypnotic or hypnoidal states and may give the framework to dreams. Yet it has its outlets, nevertheless, in bodily form by means of what Freud calls "conversion of the sum of excitation." "The sum of excitation," he says in his Studien über Hysterie, "which may not enter into psychosocial association, takes the wrong road, to bodily innervation all the more readily. The repression of the idea could only be due to an unpleasant feeling, to incompatibility between the idea to be repressed and the dominant ideational mass of the self. But the repressed idea avenges itself by becoming pathogenic." In this transformation, then, of psychical excitement into persistent bodily symptoms, or, perhaps, better in a bodily compliance or undue facility of somatic outlet of repressed psychic processes, Freud finds the essential characteristic of hysteria as contrasted with other neuroses and psychoneuroses.

British Medical Journal, 11 January 1908. p. 103

Researched by Henry Rollin. 